\title{
THE LARGE DEVIATION PRINCIPLE FOR CERTAIN SERIES
}

\author{
Miguel A. Arcones ${ }^{1}$
}

\begin{abstract}
We study the large deviation principle for stochastic processes of the form $\left\{\sum_{k=1}^{\infty} x_{k}(t) \xi_{k}\right.$ : $t \in T\}$, where $\left\{\xi_{k}\right\}_{k=1}^{\infty}$ is a sequence of i.i.d.r.v.'s with mean zero and $x_{k}(t) \in \mathbb{R}$. We present necessary and sufficient conditions for the large deviation principle for these stochastic processes in several situations. Our approach is based in showing the large deviation principle of the finite dimensional distributions and an exponential asymptotic equicontinuity condition. In order to get the exponential asymptotic equicontinuity condition, we derive new concentration inequalities, which are of independent interest.
\end{abstract}

Mathematics Subject Classification. 60F10.

Received August 5, 2003.

\section{INTRODUCTION}

We study the large deviation principle for stochastic processes of the form $\left\{\sum_{k=1}^{\infty} x_{k}(t) \xi_{k}: t \in T\right\}$, where $\left\{\xi_{k}\right\}_{k=1}^{\infty}$ is a sequence of i.i.d.r.v.'s with mean zero, $T$ is a parameter set and $x_{k}(t) \in \mathbb{R}$. Our results apply when $\log \left(\mathbb{P}\left\{\left|\xi_{1}\right| \geq t\right\}\right), t>0$, is either a convex or a concave function. In particular, we obtain necessary and sufficient conditions for the LDP of $\left\{\sum_{k=1}^{\infty} x_{k}(t) \xi_{k}: t \in T\right\}$, where $\left\{\xi_{k}\right\}$ is a sequence of symmetric i.i.d.r.v.'s such that for some $p, \tau>0$,

$$
\lim _{u \rightarrow \infty} u^{-p} \log \left(\mathbb{P}\left\{\left|\xi_{1}\right| \geq u\right\}\right)=-\tau
$$

Stochastic processes like that have been considered by several authors. If $\{X(t): t \in T\}$ is a mean zero Gaussian process such that $\sup _{t \in T}|X(t)|<\infty$ a.s., then there exists a sequence of i.i.d.r.v.'s $\left\{\xi_{k}\right\}_{k=1}^{\infty}$ with a standard normal distribution and real numbers $x_{k}(t), k \geq 1, t \in T$, such that for each $t \in T, X(t):=\sum_{k=1}^{\infty} x_{k}(t) \xi_{k}$ (see for example Prop. 2.6.1 in Kwapień and Woyczyński [10]). Hence, the considered stochastic processes are a natural generalization of Gaussian processes. Observe that for a standard normal r.v. $\xi$,

$$
\lim _{u \rightarrow \infty} u^{-2} \log (\mathbb{P}\{|\xi| \geq u\})=-2^{-1}
$$

Talagrand $[15,16]$ studied the concentration of measure and continuity of processes of the form $\left\{\sum_{k=1}^{\infty} x_{k}(t) \xi_{k}: t \in T\right\}$, where $\left\{\xi_{k}\right\}$ is a sequence of i.i.d.r.v.'s with density $c_{p} \mathrm{e}^{-|x|^{p}}, x \in \mathbb{R}$, where $p \geq 1$. Gluskin and Kwapień [8] gave tail and moment estimates for the r.v. $\sum_{k=1}^{\infty} a_{k} \xi_{k}$, where $\left\{\xi_{k}\right\}$ is a sequence of symmetric i.i.d.r.v.'s with logarithmically concave tails. Latala [11] gave tail and moment estimates for

Keywords and phrases. Large deviations, stochastic processes.

1 Department of Mathematical Sciences, Binghamton University, Binghamton, NY 13902, USA;

e-mail: arcones@math.binghamton.edu 
$\sum_{k=1}^{\infty} x_{k} \xi_{k}$, where $\left\{\xi_{k}\right\}$ is a sequence of i.i.d.r.v.'s with logarithmically concave tails and $\left\{x_{k}\right\}$ is a sequence of vectors of a Banach space. Hitczenko, Montgomery-Smith and Oleszkiewick [9] considered moment inequalities for $\sum_{i=1}^{n} \xi_{i}$, where $\left\{\xi_{i}\right\}$ is a sequence of symmetric i.i.d.r.v.'s such that $-\log \left(\mathbb{P}\left\{\left|\xi_{1}\right| \geq t\right\}\right)$ is a concave function.

We consider large deviations in the sense of Varadhan [17]. General references in large deviations are Deuschel and Stroock [7] and Dembo and Zeitouni [6]. We consider stochastic processes as random elements of $l_{\infty}(T)$, the Banach space consisting of the bounded functions on $T$ with the supremum norm. We use the following definition of large deviations for stochastic processes.

Definition 1.1. Given a sequence of stochastic processes $\left\{U_{n}(t): t \in T\right\}$, a sequence of positive numbers $\left\{\epsilon_{n}\right\}_{n=1}^{\infty}$ such that $\epsilon_{n} \rightarrow 0$, and a function $I: l_{\infty}(T) \rightarrow[0, \infty]$, we say that $\left\{U_{n}(t): t \in T\right\}$ satisfies the LDP with speed $\epsilon_{n}^{-1}$ and with rate function $I$ if:

(i) For each $0 \leq c<\infty,\left\{z \in l_{\infty}(T): I(z) \leq c\right\}$ is a compact set of $l_{\infty}(T)$.

(ii) For each set $A \subset l_{\infty}(T)$,

$$
-I\left(A^{o}\right) \leq \liminf _{n \rightarrow \infty} \epsilon_{n} \log \left(\mathbb{P}_{*}\left\{\left\{U_{n}(t): t \in T\right\} \in A\right\}\right) \leq \limsup _{n \rightarrow \infty} \epsilon_{n} \log \left(\mathbb{P}^{*}\left\{\left\{U_{n}(t): t \in T\right\} \in A\right\}\right) \leq-I(\bar{A}),
$$

where $A^{\circ}$ is the interior of $A$ and $\bar{A}$ is the closure of $A$.

It is shown in Arcones [1] that this definition is equivalent to the large deviations for the finite dimensional distributions, plus an asymptotic equicontinuity condition. This result is similar to the classical one for the weak convergence of empirical processes.

In Section 2, we present some new concentration inequalities for series processes, which are of independent interest. In Section 3, we study the large deviation principle of series processes.

$c$ will denote a finite constant, which may change from occurrence to occurrence. For $p \geq 1, l_{p}$ denotes the Banach space consisting of the sequences $\left(x_{i}\right)_{i=1}^{\infty}$ such that $\sum_{i=1}^{\infty}\left|x_{i}\right|^{p}<\infty$ endowed with the norm $\left\|\left(x_{i}\right)_{i=1}^{\infty}\right\|_{p}:=$ $\left(\sum_{i=1}^{\infty}\left|x_{i}\right|^{p}\right)^{1 / p} \cdot l_{\infty}$ denotes the Banach space consisting of the bounded sequences $\left(x_{i}\right)_{i=1}^{\infty}$ endowed with the norm $\left\|\left(x_{i}\right)_{i=1}^{\infty}\right\|_{\infty}:=\sup _{i \geq 1}\left|x_{i}\right|$. We simplify $\|x(t)\|_{p}:=\left\|\left(x_{i}(t)\right)_{i=1}^{\infty}\right\|_{p}$. We say that a function $I: S \rightarrow[0, \infty]$ is a good rate, if $\{x \in S: I(x) \leq c\}$ is a compact set, where $S$ is a topological space.

\section{Some CONCENTRATION INEQUALities FOR CERTAin SERIES PROCESSES}

It is well known that concentration inequalities play a fundamental role in the study of Gaussian processes (see for example Ledoux and Talagrand [12]). This is also so, for the considered stochastic processes. Let $\gamma_{\infty}$ be the measure on $\mathbb{R}^{\mathbb{N}}$ which is the product of $\gamma$, where $\gamma(A)=\int_{A} 2^{-1} \mathrm{e}^{-|t|} \mathrm{d} t$.

Theorem 2.1 (Talagrand [15], Th. 1). For each set $A \in \mathcal{B}\left(\mathbb{R}^{\mathbb{N}}\right)$ with $\gamma_{\infty}(A)>0$,

$$
\gamma_{\infty}\left(A+6 u^{1 / 2} B_{2}+9 u B_{1}\right) \geq 1-\left(\gamma_{\infty}(A)\right)^{-1} \exp (-u)
$$

where $B_{p}:=\left\{\left(a_{k}\right)_{k=1}^{\infty}: \sum_{k=1}^{\infty}\left|a_{k}\right|^{p} \leq 1\right\}$, for $p=1,2$. 
The previous inequality with the constants above is in Ledoux [13], Theorem 4.16.

In the case of Gaussian processes, concentration inequalities only involve the unit ball with respect to the $l_{2}$ distance. This is so, because different path properties of Gaussian processes can be characterized using the $l_{2}$ distance. However, for the considered stochastic processes, two distances are needed in general.

In this section, we present concentration inequalities for the stochastic processes $\{X(t): t \in T\}$, where $X(t):=\sum_{j=1}^{\infty} x_{j}(t) \xi_{j},\left\{\xi_{j}\right\}_{j=1}^{\infty}$ is a sequence of symmetric i.i.d.r.v.'s, $T$ is a parameter set, and $x_{j}(t) \in \mathbb{R}$. By Theorem 5.1.4 in Chow and Teicher [5], the series $\sum_{k=1}^{\infty} x_{k}(t) \xi_{k}$ converges a.s. if and only if $\sum_{k=1}^{\infty}\left(x_{k}(t)\right)^{2}<\infty$. In the considered situation, we have the following result:

Lemma 2.1. Let $\left\{\xi_{j}\right\}_{j=1}^{\infty}$ be a sequence of symmetric i.i.d.r.v.'s such that $\mathbb{P}\left[\xi_{1}=0\right]<1$. Let $T$ be a parameter set. Let $X(t)=\sum_{j=1}^{\infty} x_{j}(t) \xi_{j}, t \in T$. Suppose that $\sup _{t \in T}|X(t)|<\infty$ a.s. Then, $\left.\operatorname{Esup}_{t \in T}|X(t)|\right]<\infty$ and $\sup _{t \in T} \sum_{k=1}^{\infty} x_{k}^{2}(t)<\infty$.

Proof. Let $0<\eta<2^{-1}\left(1-\mathbb{P}\left[\xi_{1}=0\right]\right)$. Take $M>0$ such that $\mathbb{P}\left[\sup _{t \in T}|X(t)| \geq M\right] \leq \eta$. By the Lévy inequality (see for example Prop. 2.3 in Ledoux and Talagrand [12]),

$$
\sup _{t \in T} \sup _{j \geq 1} \mathbb{P}\left[\left|x_{j}(t) \xi_{j}\right| \geq M\right] \leq \mathbb{P}\left[\sup _{t \in T} \sup _{j \geq 1}\left|x_{j}(t) \xi_{j}\right| \geq M\right] \leq 2 \mathbb{P}\left[\sup _{t \in T}\left|\sum_{j=1}^{\infty} x_{j}(t) \xi_{j}\right| \geq M\right] \leq 2 \eta
$$

Hence, $\sup _{t \in T} \sup _{j \geq 1}\left|x_{j}(t)\right|<\infty$. Thus, by the Hoffmann-Jørgensen inequality (see for example Prop. 6.8 in Ledoux and Talagrand [12]), $E\left[\sup _{t \in T}|X(t)|\right]<\infty$. By the Kintchine's inequality (see for example Lem. 4.1 in Ledoux and Talagrand [12]), and the contraction principle (see for example Lem. 4.5 in Ledoux and Talagrand [12]),

$$
\begin{aligned}
2^{-1 / 2} E\left[\left|\xi_{1}\right|\right] \sup _{t \in T}\left(\sum_{k=1}^{\infty} x_{k}^{2}(t)\right)^{1 / 2} & \leq E\left[\left|\xi_{1}\right|\right] \sup _{t \in T} E\left[\left|\sum_{k=1}^{\infty} \epsilon_{k} x_{k}(t)\right|\right] \\
& \leq E\left[\left|\xi_{1}\right|\right] E\left[\sup _{t \in T}\left|\sum_{k=1}^{\infty} \epsilon_{k} x_{k}(t)\right|\right] \\
& \leq E\left[\sup _{t \in T}\left|\sum_{k=1}^{\infty} \xi_{k} x_{k}(t)\right|\right]<\infty
\end{aligned}
$$

where $\left\{\epsilon_{k}\right\}$ is a sequence of i.i.d. Rademacher r.v.'s independent of the sequence $\left\{\xi_{k}\right\}$.

We present two types of concentration inequalities according with whether the function $\Phi(t)=-\log (\mathbb{P}\{|\xi| \geq$ $t\}), t>0$, is convex or a concave.

Next, we present a concentration of measure inequality for sequences of r.v.'s satisfying the following condition:

(B.1) $\xi$ is a symmetric r.v. such that $\Phi(t)=-\log (\mathbb{P}\{|\xi| \geq t\})$, for $t>0$, is a convex increasing function.

Condition (B.1) is satisfied for many r.v.'s. In particular, (B.1) holds if $\xi$ has a symmetric distribution and $\mathbb{P}\{|\xi| \geq|t|\}=\mathrm{e}^{-c|t|^{p}}$, for each $t \geq 0$, for some $c>0$ and some $p \geq 1$. 
We will need the following lemma:

Lemma 2.2. Let $\Phi$ be as in Condition (B.1). Let

$$
\lambda(x)=\operatorname{sign}(x) \sup \{y \geq 0: \Phi(y) \leq|x|\} \text { for } x \in \mathbb{R} .
$$

Then,

(i) For each $a, b>0, \lambda(a+b) \leq \lambda(a)+\lambda(b)$.

(ii) For each $a, b \in \mathbb{R},|\lambda(a)-\lambda(b)| \leq 2 \lambda(|a-b|)$.

Proof. Since $\Phi$ is convex, $\Phi^{\prime}$ is nondecreasing. So, for each $a, b>0$,

$$
\Phi(a)+\Phi(b)=\int_{0}^{a} \Phi^{\prime}(t) \mathrm{d} t+\int_{0}^{b} \Phi^{\prime}(t) \mathrm{d} t \leq \int_{0}^{a+b} \Phi^{\prime}(t) \mathrm{d} t=\Phi(a+b) .
$$

So, (i) holds.

If either $a<0<b$, or $b<0<a$, then

$$
|\lambda(a)-\lambda(b)| \leq 2 \max (\lambda(|a|), \lambda(|b|)) \leq 2 \lambda(|a-b|) .
$$

If either $a, b<0$, or $0<a, b$, then, by (i),

$$
|\lambda(a)-\lambda(b)|=|\lambda(|a|)-\lambda(|b|)| \leq \lambda(|a-b|) .
$$

Theorem 2.2. Let $\left\{\xi_{j}\right\}_{j=1}^{\infty}$ be a sequence of symmetric i.i.d.r.v.'s satisfying Condition (B.1). Let $T$ be a parameter set. Let $X(t):=\sum_{j=1}^{\infty} x_{j}(t) \xi_{j}, t \in T$. Suppose that $\sup _{t \in T}|X(t)|<\infty$ a.s. Then, for each $0<M<\infty$,

$$
\mathbb{P}\left\{\sup _{t \in T}|X(t)| \geq M+2 L_{\Phi}(u)\right\} \leq\left(\mathbb{P}\left\{\sup _{t \in T}|X(t)|<M\right\}\right)^{-1} \mathrm{e}^{-u}
$$

where

$$
L_{\Phi}(u):=\sup \left\{\sum_{k=1}^{\infty}\left|x_{k}(t)\right|\left(\left|a_{k}\right|+\left|b_{k}\right|\right): \sum_{k=1}^{\infty}\left(\Phi\left(a_{k}\right)\right)^{2} \leq 36 u, \sum_{k=1}^{\infty} \Phi\left(b_{k}\right) \leq 9 u, t \in T\right\} .
$$

Proof. Let $\left\{Y_{i}\right\}_{i=1}^{\infty}$ be a sequence of symmetric i.i.d.r.v.'s with $\mathbb{P}\left\{\left|Y_{i}\right| \geq t\right\}=\mathrm{e}^{-t}$, for each $t \geq 0$ and each $i \geq 1$. Let $\lambda$ be as in (2.2). Then, for each $x, y \geq 0, \Phi(y) \leq x$ iff $y \leq \lambda(x)$. By the continuity of the function $\Phi$, we also have that $\Phi(\lambda(x))=x$, for each $x \geq 0$. Hence, we have that for each $t \geq 0$,

$$
\mathbb{P}\left\{\left|\lambda\left(Y_{i}\right)\right| \geq t\right\}=\mathbb{P}\left\{\lambda\left(\left|Y_{i}\right|\right) \geq t\right\}=\mathbb{P}\left\{\left|Y_{i}\right| \geq \Phi(t)\right\}=\mathrm{e}^{-\Phi(t)}=\mathbb{P}\left\{\left|\xi_{i}\right| \geq t\right\}
$$

Therefore, $\xi_{i}$ and $\lambda\left(Y_{i}\right)$ have the same distribution.

Let $A=\left\{\left(y_{i}\right)_{i=1}^{\infty}: \sup _{t \in T}\left|\sum_{k=1}^{\infty} x_{k}(t) \lambda\left(y_{i}\right)\right|<M\right\}$. By $(2.1)$

$$
\mathbb{P}\left\{\left(Y_{i}\right)_{i=1}^{\infty} \notin A+6 u^{1 / 2} B_{2}+9 u B_{1}\right\} \leq\left(\mathbb{P}\left\{\left(Y_{i}\right)_{i=1}^{\infty} \in A\right\}\right)^{-1} \mathrm{e}^{-u}
$$


So, it suffices to show that if $\left(y_{i}\right)_{i=1}^{\infty} \in A+6 u^{1 / 2} B_{2}+9 u B_{1}$, then

$$
\sup _{t \in T}\left|\sum_{i=1}^{\infty} x_{i}(t) \lambda\left(y_{i}\right)\right|<M+2 L_{\Phi}(u) .
$$

We have that there are $\left(a_{i}\right)_{i=1}^{\infty} \in A,\left(b_{i}\right)_{i=1}^{\infty} \in B_{2}$, and $\left(c_{i}\right)_{i=1}^{\infty} \in B_{1}$, such that for each $i \geq 1, y_{i}=a_{i}+6 u^{1 / 2} b_{i}+$ $9 u c_{i}$. By Lemma 2.2,

$$
\begin{aligned}
\sup _{t \in T}\left|\sum_{i=1}^{\infty} x_{i}(t) \lambda\left(a_{i}+6 u^{1 / 2} b_{i}+9 u c_{i}\right)\right| & \leq \sup _{t \in T}\left|\sum_{i=1}^{\infty} x_{i}(t) \lambda\left(a_{i}\right)\right|+2 \sup _{t \in T} \sum_{i=1}^{\infty}\left|x_{i}(t)\right| \lambda\left(\left|6 u^{1 / 2} b_{i}+9 u c_{i}\right|\right) \\
& \leq M+2 \sup _{t \in T} \sum_{i=1}^{\infty}\left|x_{i}(t)\right| \lambda\left(6 u^{1 / 2}\left|b_{i}\right|\right)+2 \sup _{t \in T} \sum_{i=1}^{\infty}\left|x_{i}(t)\right| \lambda\left(9 u\left|c_{i}\right|\right) \\
& \leq M+2 L_{\Phi}(u) .
\end{aligned}
$$

Because,

$$
\sum_{i=1}^{\infty}\left(\Phi\left(\lambda\left(6 u^{1 / 2}\left|b_{i}\right|\right)\right)\right)^{2} \leq \sum_{i=1}^{\infty} 36 u b_{i}^{2} \leq 36 u
$$

and

$$
\sum_{i=1}^{\infty} \Phi\left(\lambda\left(9 u\left|c_{i}\right|\right)\right) \leq \sum_{i=1}^{\infty} 9 u\left|c_{i}\right| \leq 9 u
$$

Corollary 2.1. Assume the notation and conditions in the previous theorem. Suppose also that there are constants $\tau>0$ and $p \geq 1$ such that $\tau \Phi(x) \geq \max \left(|x|^{p},|x|\right)$, for each $x>0$. Then,

(i) If $p \geq 2$, then, for each $0<M<\infty$ and each $u>0$,

$$
\mathbb{P}\left\{\sup _{t \in T}|X(t)| \geq M+2 \sup _{t \in T}\|x(t)\|_{q}\left(36 u \tau^{2}\right)^{1 / p}+2 \sup _{t \in T}\|x(t)\|_{q}(9 \tau u)^{1 / p}\right\} \leq\left(\mathbb{P}\left\{\sup _{t \in T}|X(t)|<M\right\}\right)^{-1} \mathrm{e}^{-u} .
$$

(ii) If $2 \geq p \geq 1$, then, for each $0<M<\infty$ and each $u>0$,

$$
\mathbb{P}\left\{\sup _{t \in T}|X(t)| \geq M+12 \sup _{t \in T}\|x(t)\|_{2} \tau u^{1 / 2}+2 \sup _{t \in T}\|x(t)\|_{q}(9 \tau u)^{1 / p}\right\} \leq\left(\mathbb{P}\left\{\sup _{t \in T}|X(t)|<M\right\}\right)^{-1} \mathrm{e}^{-u}
$$

Proof. By Theorem 2.2, we need to prove that if $p \geq 2$,

$$
L_{\Phi}(u) \leq \sup _{t \in T}\|x(t)\|_{q}\left(36 \tau^{2} u\right)^{1 / p}+\sup _{t \in T}\|x(t)\|_{q}(9 \tau u)^{1 / p} ;
$$

and if $1 \leq p<2$,

$$
L_{\Phi}(u) \leq 6 \sup _{t \in T}\|x(t)\|_{2} \tau u^{1 / 2}+\sup _{t \in T}\|x(t)\|_{q}(9 \tau u)^{1 / p} .
$$

Suppose that

$$
\sum_{k=1}^{\infty}\left(\Phi\left(a_{k}\right)\right)^{2} \leq 36 u \text { and } \sum_{k=1}^{\infty} \Phi\left(b_{k}\right) \leq 9 u
$$


If $p \geq 2$,

$$
\begin{aligned}
\sum_{k=1}^{\infty}\left|x_{k}(t) \| a_{k}\right| & \leq\|x(t)\|_{q}\left(\sum_{k=1}^{\infty}\left|a_{k}\right|^{p}\right)^{1 / p} \leq\|x(t)\|_{q}\left(\sum_{k=1}^{\infty}\left(\max \left(\left|a_{k}\right|,\left|a_{k}\right|^{p}\right)\right)^{2}\right)^{1 / p} \\
& \leq\|x(t)\|_{q} \tau^{2 / p}\left(\sum_{k=1}^{\infty}\left(\Phi\left(\left|a_{k}\right|\right)\right)^{2}\right)^{1 / p} \leq\|x(t)\|_{q}\left(36 \tau^{2} u\right)^{1 / p} .
\end{aligned}
$$

If $1 \leq p<2$,

$$
\begin{aligned}
\sum_{k=1}^{\infty}\left|x_{k}(t) \| a_{k}\right| & \leq\|x(t)\|_{2}\left(\sum_{k=1}^{\infty}\left|a_{k}\right|^{2}\right)^{1 / 2} \\
& \leq\|x(t)\|_{2} \tau\left(\sum_{k=1}^{\infty}\left(\Phi\left(\left|a_{k}\right|\right)\right)^{2}\right)^{1 / 2} \leq 6\|x(t)\|_{2} \tau u^{1 / 2} .
\end{aligned}
$$

We have that

$$
\begin{aligned}
\sum_{k=1}^{\infty}\left|x_{k}(t) \| b_{k}\right| & \leq\|x(t)\|_{q}\left(\sum_{j=1}^{\infty}\left|b_{k}\right|^{p}\right)^{1 / p} \\
& \leq\|x(t)\|_{q} \tau^{1 / p}\left(\sum_{j=1}^{\infty} \Phi\left(b_{k}\right)\right)^{1 / p} \leq\|x(t)\|_{q}(9 \tau u)^{1 / p}
\end{aligned}
$$

It follows from the previous corollary that for $u$ large enough,

$$
\mathbb{P}\left\{\sup _{t \in T}|X(t)| \geq u\right\} \leq 2 \exp \left(-c u^{p} \sup _{t \in T}\|x(t)\|_{q}^{-p}\right)
$$

where $c$ is some positive constant.

In the situation of the previous corollary the asymptotic exponential order of the tail of $\sup _{t \in T}|X(t)|$ is the same as the asymptotic exponential order of $\xi_{1}$. In Theorem 3.4, we give an example of r.v.'s for which the asymptotic exponential order of the tail of $\sup _{t \in T}|X(t)|$ is bigger than the asymptotic exponential order of $\xi_{1}$.

Next, we consider the case when $-\log (\mathbb{P}\{|\xi| \geq t\}), t \geq 0$, is a concave function. We consider the following condition:

(B.2) $\xi$ is a symmetric r.v. such that:

(i) $\Phi(t)=-\log (\mathbb{P}\{|\xi| \geq t\}), t>0$ is a concave increasing function.

(ii) There exists a constant $\tau>0$ such that for each $a \geq 0,2 \Phi(a) \leq \Phi(\tau a)$.

If for some $\eta>0$ and some $1 \geq p>0, \mathbb{P}\{|\xi| \geq t\}=\exp \left(-\eta t^{p}\right)$, for each $t \geq 0$, then (B.2) holds.

Condition (B.2) (iii) imposes a sort of polynomical growth. If $\mathbb{P}\{|\xi| \geq t\}=\frac{1}{t+1}$, for each $t \geq 0$, then $\Phi(t)=-\log (\mathbb{P}\{|Z| \geq t\})=\ln (t+1), t>0$, is a concave function. But, (B.2) (ii) does not hold. For each $\tau>0$, $\inf _{a>0} \frac{\Phi(\tau a)}{\Phi(a)}=1$. 
Theorem 2.3. Let $\left\{\xi_{j}\right\}_{j=1}^{\infty}$ be a sequence of i.i.d.r.v.'s satisfying Condition (B.2). Let T be a parameter set. Let $X(t):=\sum_{j=1}^{\infty} x_{j}(t) \xi_{j}, t \in T$, where $\sum_{j=1}^{\infty}\left|x_{j}(t)\right|^{2}<\infty$ for each $t \in T$. Then, for each $u>0$,

$$
\begin{aligned}
\mathbb{P}\left\{\sup _{t \in T}|X(t)| \geq\right. & M\left(4+68 \tau^{2} u^{1 / 2}+102 \tau^{2} u\right) \\
& +\sup _{t \in T}\|x(t)\|_{2}\left(\lambda\left(6 u^{1 / 2}\right)+12 \tau u^{1 / 2}+12(\tau+1) u^{1 / 2} \lambda^{\prime}\left(6 u^{1 / 2}+9 u\right)\right) \\
& \left.+\sup _{t \in T}\|x(t)\|_{\infty}\left(\lambda(9 u)+18 \tau u \lambda^{\prime}(1)+18 \tau u \lambda^{\prime}\left(6 u^{1 / 2}+9 u\right)\right)\right\} \\
\leq & 2 \mathrm{e}^{-u},
\end{aligned}
$$

where $M=E\left[\sup _{t \in T}|X(t)|\right]$.

Proof. We proceed as in Theorem 2.2. Let $\left\{Y_{i}\right\}_{i=1}^{\infty}$ be a sequence of symmetric i.i.d.r.v.'s with $\mathbb{P}\left\{\left|Y_{i}\right| \geq t\right\}=\mathrm{e}^{-t}$, for each $t \geq 0$ and each $i \geq 1$. Then, $\xi_{i}$ has the distribution of $\lambda\left(Y_{i}\right)$, where $\lambda$. By Condition (B.2), $\lambda$ is a convex function in $[0, \infty)$, with $\lambda(0)=0$ and for each $a \geq 0$,

$$
\lambda^{\prime}(2 a) \leq \tau \lambda^{\prime}(a) .
$$

Let

$$
A=\left\{\left(a_{k}\right)_{k=1}^{\infty}: \sup _{t \in T}\left|\sum_{k=1}^{\infty} x_{k}(t) \lambda\left(a_{k}\right)\right|<4 M, \sup _{t \in T} \sum_{k=1}^{\infty} x_{k}^{2}(t)\left(\lambda\left(a_{k}\right)\right)^{2}<32 M^{2}\right\} .
$$

We claim that

$$
\mathbb{P}\left\{\left(Y_{k}\right)_{k=1}^{\infty} \in A\right) \geq 1 / 2 .
$$

By the Chebyshev inequality,

$$
\mathbb{P}\left\{\sup _{t \in T}\left|\sum_{k=1}^{\infty} x_{k}(t) \lambda\left(Y_{k}\right)\right| \geq 4 M\right\} \leq 1 / 4
$$

By the Kintchine inequality,

$$
\begin{aligned}
\mathbb{P}\left\{\sup _{t \in T} \sum_{k=1}^{\infty} x_{k}^{2}(t)\left(\lambda\left(Y_{k}\right)\right)^{2} \geq 32 M^{2}\right\} & \leq 32^{-1 / 2} M^{-1} E\left[\sup _{t \in T}\left(\sum_{k=1}^{\infty} x_{k}^{2}(t)\left(\lambda\left(Y_{k}\right)\right)^{2}\right)^{1 / 2}\right] \\
& =32^{-1 / 2} M^{-1} E\left[\sup _{t \in T}\left(E_{\epsilon}\left[\left(\sum_{k=1}^{\infty} \epsilon_{k} x_{k}(t) \lambda\left(Y_{k}\right)\right)^{2}\right]\right)^{1 / 2}\right] \\
& \leq 2^{-2} M^{-1} E\left[\sup _{t \in T} E_{\epsilon}\left[\left|\sum_{k=1}^{\infty} \epsilon_{k} x_{k}(t) \lambda\left(Y_{k}\right)\right|\right]\right] \\
& \leq 2^{-2} M^{-1} E\left[\sup _{t \in T}\left|\sum_{k=1}^{\infty} \epsilon_{k} x_{k}(t) \lambda\left(Y_{k}\right)\right|\right]=2^{-2},
\end{aligned}
$$

where $\left\{\epsilon_{k}\right\}$ is a sequence of i.i.d. Rademacher r.v.'s independent of the sequence $\left\{\xi_{k}\right\}$. Therefore, $\gamma_{\infty}(A) \geq 1 / 2$. 
By (2.1), it suffices to show that if $\left(y_{i}\right)_{i=1}^{\infty} \in A+6 u^{1 / 2} B_{2}+9 u B_{1}$, then

$$
\begin{aligned}
\sup _{t \in T}\left|\sum_{i=1}^{\infty} x_{i}(t) \lambda\left(y_{i}\right)\right|< & M\left(4+68 \tau^{2} u^{1 / 2}+102 \tau^{2} u\right) \\
& +\sup _{t \in T}\|x(t)\|_{2}\left(\lambda\left(6 u^{1 / 2}\right)+12 \tau u^{1 / 2}+12(\tau+1) u^{1 / 2} \lambda^{\prime}\left(6 u^{1 / 2}+9 u\right)\right) \\
& +\sup _{t \in T}\|x(t)\|_{\infty}\left(\lambda(9 u)+18 \tau u \lambda^{\prime}(1)+18 \tau u \lambda^{\prime}\left(6 u^{1 / 2}+9 u\right)\right) .
\end{aligned}
$$

We have that there are $\left(a_{i}\right)_{i=1}^{\infty} \in A,\left(b_{i}\right)_{i=1}^{\infty} \in B_{2}$, and $\left(c_{i}\right)_{i=1}^{\infty} \in B_{1}$, such that for each $i \geq 1, y_{i}=a_{i}+6 u^{1 / 2} b_{i}+$ $9 u c_{i}$. Since $\lambda^{\prime}(t)$ is an even function an it is nondecreasing in $[0, \infty)$, for each $a, b \in \mathbb{R}$, with $|b| \leq|a|$,

$$
|\lambda(a+b)-\lambda(a)| \leq|b| \lambda^{\prime}(|a|+|b|)
$$

and $|\lambda(b)| \leq|b| \lambda^{\prime}(|b|)$. So, for each $a, b \in \mathbb{R}$,

$$
|\lambda(a+b)-\lambda(a)-\lambda(b)| \leq 2 \min (|a|,|b|) \lambda^{\prime}(|a|+|b|) .
$$

Using (2.7), for each $a, b \in \mathbb{R}$,

$$
\left|\lambda^{\prime}(a+b)\right| \leq \lambda^{\prime}(|a|+|b|) \leq \lambda^{\prime}(2 \max (|a|,|b|)) \leq \tau \lambda^{\prime}(\max (|a|,|b|)) \leq \tau\left(\lambda^{\prime}(|a|)+\lambda^{\prime}(|b|)\right) .
$$

Using the previous inequality and (2.8), we get that for each $a, b \in \mathbb{R}$,

$$
|\lambda(a+b)-\lambda(a)-\lambda(b)| \leq 2 \tau \min (|a|,|b|)\left(\lambda^{\prime}(|a|)+\lambda^{\prime}(|b|)\right) .
$$

Using (2.8) and (2.9), for each $i \geq 1$,

$$
\begin{aligned}
\mid \lambda\left(a_{i}+6 u^{1 / 2} b_{i}+\right. & \left.9 u c_{i}\right)-\lambda\left(a_{i}\right)-\lambda\left(6 u^{1 / 2} b_{i}\right)-\lambda\left(9 u c_{i}\right)|\leq| \lambda\left(a_{i}+6 u^{1 / 2} b_{i}+9 u c_{i}\right) \\
- & \lambda\left(a_{i}\right)-\lambda\left(6 u^{1 / 2} b_{i}+9 u c_{i}\right)|+| \lambda\left(6 u^{1 / 2} b_{i}+9 u c_{i}\right)-\lambda\left(6 u^{1 / 2} b_{i}\right)-\lambda\left(9 u c_{i}\right) \mid \\
& \leq 2 \tau\left(6 u^{1 / 2}\left|b_{i}\right|+9 u\left|c_{i}\right|\right) \lambda^{\prime}\left(\left|a_{i}\right|\right)+2 \tau\left(6 u^{1 / 2}\left|b_{i}\right|+9 u\left|c_{i}\right|\right) \lambda^{\prime}\left(6 u^{1 / 2}+9 u\right) \\
+ & +12 u^{1 / 2}\left|b_{i}\right| \lambda^{\prime}\left(6 u^{1 / 2}+9 u\right)
\end{aligned}
$$

Since $\lambda^{\prime}$ is nondecreasing in $[0, \infty)$, for each $a>0$,

$$
\lambda(2 a)=\int_{0}^{2 a} \lambda^{\prime}(t) \mathrm{d} t \geq \int_{a}^{2 a} \lambda^{\prime}(t) \mathrm{d} t \geq a \lambda^{\prime}(a) .
$$

By Condition (B.1), for each $a>0, \lambda(2 a) \leq \tau \lambda(a)$. So, for each for each $a>0, a \lambda^{\prime}(a) \leq \tau \lambda(a)$. Hence, for each $a \in \mathbb{R}$

By (2.10) and (2.11), for each $i \geq 1$,

$$
\lambda^{\prime}(|a|) \leq \lambda^{\prime}(1)+\tau \lambda(|a|) .
$$

$$
\begin{aligned}
\mid \lambda\left(a_{i}+6\right. & \left.u^{1 / 2} b_{i}+9 u c_{i}\right)-\lambda\left(a_{i}\right)-\lambda\left(6 u^{1 / 2} b_{i}\right)-\lambda\left(9 u c_{i}\right)\left|\leq 12 \tau \lambda^{\prime}(1) u^{1 / 2}\right| b_{i}\left|+18 \tau \lambda^{\prime}(1) u\right| c_{i} \mid \\
& +12 \tau^{2} u^{1 / 2}\left|b_{i}\right| \lambda\left(\left|a_{i}\right|\right)+18 \tau^{2} u\left|c_{i}\right| \lambda\left(\left|a_{i}\right|\right)+12(\tau+1) u^{1 / 2}\left|b_{i}\right| \lambda^{\prime}\left(6 u^{1 / 2}+9 u\right)+18 \tau u\left|c_{i}\right| \lambda^{\prime}\left(6 u^{1 / 2}+9 u\right) .
\end{aligned}
$$


These estimations imply that for $\left(a_{i}\right)_{i=1}^{\infty} \in A,\left(b_{i}\right)_{i=1}^{\infty} \in B_{2}$, and $\left(c_{i}\right)_{i=1}^{\infty} \in B_{1}$,

$$
\begin{aligned}
& \left|\sum_{i=1}^{\infty} x_{i}(t) \lambda\left(a_{i}+6 u^{1 / 2} b_{i}+9 u c_{i}\right)\right| \leq\left|\sum_{i=1}^{\infty} x_{i}(t) \lambda\left(a_{i}\right)\right|+\left|\sum_{i=1}^{\infty} x_{i}(t) \lambda\left(6 u^{1 / 2} b_{i}\right)\right|+\left|\sum_{i=1}^{\infty} x_{i}(t) \lambda\left(9 u c_{i}\right)\right| \\
& +12 \tau \lambda^{\prime}(1) u^{1 / 2} \sum_{i=1}^{\infty}\left|x_{i}(t)\right|\left|b_{i}\right|+18 \tau \lambda^{\prime}(1) u \sum_{i=1}^{\infty}\left|x_{i}(t)\right|\left|c_{i}\right| \\
& +12 \tau^{2} u^{1 / 2} \sum_{i=1}^{\infty}\left|x_{i}(t)\right|\left|b_{i}\right| \lambda\left(\left|a_{i}\right|\right)+18 \tau^{2} u \sum_{i=1}^{\infty}\left|x_{i}(t) \| c_{i}\right| \lambda\left(\left|a_{i}\right|\right) \\
& +12(\tau+1) u^{1 / 2} \lambda^{\prime}\left(6 u^{1 / 2}+9 u\right) \sum_{i=1}^{\infty}\left|x_{i}(t)\right|\left|b_{i}\right| \\
& +18 \tau u \lambda^{\prime}\left(6 u^{1 / 2}+9 u\right) \sum_{i=1}^{\infty}\left|x_{i}(t)\right|\left|c_{i}\right| \\
& \leq\left|\sum_{i=1}^{\infty} x_{i}(t) \lambda\left(a_{i}\right)\right|+\sum_{i=1}^{\infty}\left|x_{i}(t)\right| b_{i}\left|\lambda\left(6 u^{1 / 2}\right)\right|+\sum_{i=1}^{\infty}\left|x_{i}(t)\right| c_{i}|\lambda(9 u)| \\
& +12 \tau u^{1 / 2} \lambda^{\prime}(1) \sum_{i=1}^{\infty}\left|x_{i}(t)\right|\left|b_{i}\right|+18 \tau u \lambda^{\prime}(1) \sum_{i=1}^{\infty}\left|x_{i}(t)\right|\left|c_{i}\right| \\
& +12 \tau^{2} u^{1 / 2} \sum_{i=1}^{\infty}\left|x_{i}(t)\right| \lambda\left(\left|a_{i}\right|\right)+18 \tau^{2} u \sum_{i=1}^{\infty}\left|x_{i}(t)\right| \lambda\left(\left|a_{i}\right|\right) \\
& +12(\tau+1) u^{1 / 2} \lambda^{\prime}\left(6 u^{1 / 2}+9 u\right) \sum_{i=1}^{\infty}\left|x_{i}(t)\right|\left|b_{i}\right| \\
& +18 \tau u \lambda^{\prime}\left(6 u^{1 / 2}+9 u\right) \sum_{i=1}^{\infty}\left|x_{i}(t)\right|\left|c_{i}\right| \\
& \leq 4 M+\|x(t)\|_{2} \lambda\left(6 u^{1 / 2}\right)+\|x(t)\|_{\infty} \lambda(9 u)+12 \tau u^{1 / 2} \lambda^{\prime}(1)\|x(t)\|_{2} \\
& +18 \tau u \lambda^{\prime}(1)\|x(t)\|_{\infty}+68 \tau^{2} u^{1 / 2} M+102 \tau^{2} u M \\
& +12(\tau+1) u^{1 / 2} \lambda^{\prime}\left(6 u^{1 / 2}+9 u\right)\|x(t)\|_{2}+18 \tau u \lambda^{\prime}\left(6 u^{1 / 2}+9 u\right)\|x(t)\|_{\infty} \\
& =M\left(4+68 \tau^{2} u^{1 / 2}+102 \tau^{2} u\right) \\
& +\|x(t)\|_{2}\left(\lambda\left(6 u^{1 / 2}\right)+12 \tau u^{1 / 2} \lambda^{\prime}(1)+12(\tau+1) u^{1 / 2} \lambda^{\prime}\left(6 u^{1 / 2}+9 u\right)\right) \\
& +\|x(t)\|_{\infty}\left(\lambda(9 u)+18 \tau u \lambda^{\prime}(1)+18 \tau u \lambda^{\prime}\left(6 u^{1 / 2}+9 u\right)\right),
\end{aligned}
$$

where we have used that for each $0 \leq t \leq 1$ and $x \geq 0, \lambda(t x) \leq t \lambda(x)$. This follows from the convexity of $\lambda$ in $[0, \infty)$. This implies the claim of the theorem.

The term of bigger order, as $u \rightarrow \infty$, inside the probability in (2.6) is $\lambda(9 u)$. So, the previous theorem gives that there exists a constant $c$ such that for $u$ large enough,

$$
\mathbb{P}\left\{\sup _{t \in T}|X(t)| \geq c \sup _{t \in T}\|x(t)\|_{\infty} \lambda(u)\right\} \leq 2 \mathrm{e}^{-u} .
$$




\section{THE LDP FOR CERTAIN SERIES PROCESSES}

Our goal is to study the LDP of $\left\{\sum_{k=1}^{\infty} x_{k}(t) \xi_{k}: t \in T\right\}$, where $\left\{\xi_{k}\right\}_{k=1}^{\infty}$ is a sequence of i.i.d.r.v.'s with mean zero, $T$ is a parameter set and $x_{k}(t) \in \mathbb{R}$. We present results on the order of generality of the r.v.'s considered. First, we will study the LDP for $\left\{n^{-1} \xi\right\}$, where $\xi$ is a r.v. Then, we will study the LDP for $\left\{\sum_{k=1}^{\infty} x_{k} \xi_{k}\right\}$, where $\left\{x_{k}\right\}$ is a sequence of real numbers. Finally, we will consider the LDP for $\left\{\sum_{k=1}^{\infty} x_{k}(t) \xi_{k}: t \in T\right\}$.

In order to obtain the LDP for $\left\{n^{-1} \xi\right\}$, it is needed to impose that the tail of the r.v. $\xi$ is regularly varying. We refer to regular variation to Bingham, Goldie and Teugels [4]. We consider the following condition:

(A.1) $-\log (\mathbb{P}\{|\xi| \geq t\})$ is regularly varying at infinity with order $p>0$ and for some $0<c_{1}, c_{2} \leq \infty$,

$$
\lim _{u \rightarrow \infty} \frac{\log (\mathbb{P}\{\xi \leq-u\})}{\log (\mathbb{P}\{|\xi| \geq u\})}=c_{1}
$$

and

$$
\lim _{\lambda \rightarrow \infty} \frac{\log (\mathbb{P}\{\xi \geq u\})}{\log (\mathbb{P}\{|\xi| \geq u\})}=c_{2} .
$$

Since for each $u>0, \mathbb{P}\{\xi \leq-u\} \leq \mathbb{P}\{|\xi| \geq u\}$ and $\mathbb{P}\{\xi \geq u\} \leq \mathbb{P}\{|\xi| \geq u\}, c_{1} \geq 1$ and $c_{2} \geq 1$. It is easy to see that $\min \left(c_{1}, c_{2}\right)=1$. Under Condition (A.1), $\left\{n^{-1} \xi\right\}$ satisfies the LDP with speed $\epsilon_{n}^{-1}:=-\log (\mathbb{P}\{|\xi| \geq n\})$ and rate function

$$
\psi(t)= \begin{cases}c_{1}|t|^{p} & \text { if } t<0 \\ 0 & \text { if } t=0 \\ c_{2}|t|^{p} & \text { if } t>0\end{cases}
$$

Under minimal conditions if $\left\{n^{-1} \xi\right\}$ satisfies the LDP, Condition (A.1) holds:

Theorem 3.1. Let $\xi$ be a r.v. and let $\left\{\epsilon_{n}\right\}_{n=1}^{\infty}$ be a sequence of positive numbers converging to zero. Suppose that:

(i) $\left\{n^{-1} \xi\right\}$ satisfies the LDP with speed $\epsilon_{n}^{-1}$ and a good rate function $\psi$.

(ii) $\psi$ is continuous in $\mathbb{R}-\{0\}$.

(iii) There exists $0<t_{0}<\infty$ such that $0<K\left(t_{0}\right)<\infty$, where $K(t)=\inf \{\psi(s):|s| \geq t\}$, for $t>0$.

Then, Condition (A.1) holds and $\psi$ is as in (3.1) for some $p>0$ and some $0<c_{1}, c_{2} \leq \infty$.

Proof. Let $J(t)=\inf \{\psi(s): s \geq t\}$, if $t>0$ and $J(t)=\inf \{\psi(s): s \leq t\}$, if $t<0$. Then, for each $t>0$, $K(t)=\min (J(t), J(-t))$. By Conditions (i) and (ii), $j$ and $K$ are continuous functions on $\mathbb{R}-\{0\}$. Hence, for each $t>0$,

$$
\lim _{n \rightarrow \infty} \epsilon_{n} \log (\mathbb{P}\{\xi \geq t n\})=-J(t)
$$

and for each $t<0$,

$$
\lim _{n \rightarrow \infty} \epsilon_{n} \log (\mathbb{P}\{\xi \leq t n\})=-J(t) .
$$

Besides for each $t>0$,

$$
\lim _{n \rightarrow \infty} \epsilon_{n} \log (\mathbb{P}\{|\xi| \geq t n\})=-K(t) .
$$

By the Karamata theorem (see for example Th. 1.10.2 in Bingham, Goldie and Teugels [4]), $-\log (\mathbb{P}\{|\xi| \geq t\}$ ) is regularly varying at infinity with order $p \geq 0$ and there is a $0<c<\infty$ such that for each $t>0, K(t)=c t^{p}$. Since $\psi$ is a good rate, $\lim _{|t| \rightarrow \infty} \psi(t)=\infty$ and $p>0$.

We claim that

$$
J(t)= \begin{cases}c_{1}|t|^{p} & \text { if } t<0 \\ c_{2}|t|^{p} & \text { if } t>0,\end{cases}
$$


where $c_{1}=\frac{c J\left(-t_{0}\right)}{K\left(t_{0}\right)}$ and $c_{2}=\frac{c J\left(t_{0}\right)}{K\left(t_{0}\right)}$. We have that

$$
\lim _{n \rightarrow \infty} \frac{\log \left(\mathbb{P}\left\{\xi \geq t_{0} n\right\}\right)}{\log \left(\mathbb{P}\left\{|\xi| \geq t_{0} n\right\}\right)}=\frac{J\left(t_{0}\right)}{K\left(t_{0}\right)}
$$

This limit and the regular variation of $-\log (\mathbb{P}\{|\xi| \geq t\})$, implies that

$$
\lim _{u \rightarrow \infty} \frac{\log (\mathbb{P}\{\xi \geq u\})}{\log (\mathbb{P}\{|\xi| \geq u\})}=\frac{J\left(t_{0}\right)}{K\left(t_{0}\right)}
$$

So, for each $t>0$,

$$
\epsilon_{n} \log (\mathbb{P}\{\xi \geq t n\})=\epsilon_{n} \log (\mathbb{P}\{|\xi| \geq t n\}) \frac{\log (\mathbb{P}\{\xi \geq t n\})}{\log (\mathbb{P}\{|\xi| \geq t n\})} \rightarrow-c_{2}|t|^{p}
$$

Similarly, for each $t<0$,

$$
\epsilon_{n} \log (\mathbb{P}\{\xi \leq t n\}) \rightarrow-c_{1}|t|^{p} .
$$

So, $J$ is as in (3.2). The limits (3.3) and (3.4) imply that $\psi$ is as in (3.1).

Next, we consider the LDP for the series $\sum_{k=1}^{\infty} x_{k} \xi_{k}$, where $\left\{\xi_{k}\right\}$ is a sequence of i.i.d.r.v.'s with mean zero and finite second moment and $\left\{x_{k}\right\}$ is a sequence of real numbers. To determine the rate function of the LDP of $\left\{n^{-1} \sum_{k=1}^{\infty} x_{k} \xi_{k}\right\}$, we will use the following lemma:

Lemma 3.1. Let $\psi$ be as in (3.1), where $p>0,0<c_{1}, c_{2} \leq \infty$ and $\min \left(c_{1}, c_{2}\right)<\infty$. Let $\left\{x_{k}\right\}_{k=1}^{\infty}$ be a sequence of real numbers such that $\sum_{k=1}^{\infty} x_{k}^{2}<\infty$. If $p \geq 2$, assume also that $\sum_{k=1}^{\infty}\left|x_{k}\right|^{q}<\infty$, where $\frac{1}{p}+\frac{1}{q}=1$. Let

$$
I(z)=\inf \left\{\sum_{j=1}^{\infty} \psi\left(u_{j}\right): \sum_{j=1}^{\infty} u_{j} x_{j}=z\right\}
$$

If $p>1$, then

$$
I(z)= \begin{cases}|z|^{p}\left(c_{1}^{-q / p} \sum_{i \in A_{2}}\left|x_{i}\right|^{q}+c_{2}^{-q / p} \sum_{i \in A_{1}}\left|x_{i}\right|^{q}\right)^{-p / q} & \text { if } z<0 \\ |z|^{p}\left(c_{1}^{-q / p} \sum_{i \in A_{1}}\left|x_{i}\right|^{q}+c_{2}^{-q / p} \sum_{i \in A_{2}}\left|x_{i}\right|^{q}\right)^{-p / q} & \text { if } z \geq 0\end{cases}
$$

where $A_{1}=\left\{i \geq 1: x_{i}<0\right\}$ and $A_{2}=\left\{i \geq 1: x_{i}>0\right\}$.

If $1 \geq p>0$, then

$$
I(z)= \begin{cases}|z|^{p}\left(\max \left(\sup _{i \in A_{2}} c_{1}^{-1}\left|x_{i}\right|^{p}, \sup _{i \in A_{1}} c_{2}^{-1}\left|x_{i}\right|^{p}\right)\right)^{-1} & \text { if } z<0 \\ |z|^{p}\left(\max \left(\sup _{i \in A_{1}} c_{1}^{-1}\left|x_{i}\right|^{p}, \sup _{i \in A_{2}} c_{2}^{-1}\left|x_{i}\right|^{p}\right)\right)^{-1} & \text { if } z \geq 0 .\end{cases}
$$


Proof. Assume that $p>1, c_{1}, c_{2}<\infty$ and $\sum_{j=1}^{\infty} u_{j} x_{j}=z>0$. By he Hölder inequality,

$$
\begin{aligned}
z= & \sum_{i \in A_{1}} x_{i} u_{i}+\sum_{i \in A_{2}} x_{i} u_{i} \\
\leq & \left(\sum_{i \in A_{1}}\left|x_{i}\right|^{q}\right)^{1 / q}\left(\sum_{i \in A_{1}}\left|u_{i}\right|^{p}\right)^{1 / p}+\left(\sum_{i \in A_{2}}\left|x_{i}\right|^{q}\right)^{1 / q}\left(\sum_{i \in A_{2}}\left|u_{i}\right|^{p}\right)^{1 / p} \\
= & \left(c_{1}^{-q / p} \sum_{i \in A_{1}}\left|x_{i}\right|^{q}\right)^{1 / q}\left(c_{1} \sum_{i \in A_{1}}\left|u_{i}\right|^{p}\right)^{1 / p} \\
& +\left(c_{2}^{-q / p} \sum_{i \in A_{2}}\left|x_{i}\right|^{q}\right)^{1 / q}\left(c_{2} \sum_{i \in A_{2}}\left|u_{i}\right|^{p}\right)^{1 / p} \\
\leq & \left(c_{1}^{-q / p} \sum_{i \in A_{1}}\left|x_{i}\right|^{q}+c_{2}^{-q / p} \sum_{i \in A_{2}}\left|x_{i}\right|^{q}\right)^{1 / q} \\
& \times\left(c_{1} \sum_{i \in A_{1}}\left|u_{i}\right|^{p}+c_{2} \sum_{i \in A_{2}}\left|u_{i}\right|^{p}\right)^{1 / p} \\
= & \left(c_{1}^{-q / p} \sum_{i \in A_{1}}\left|x_{i}\right|^{q}+c_{2}^{-q / p} \sum_{i \in A_{2}}\left|x_{i}\right|^{q}\right)^{1 / q}\left(\sum_{i=1}^{\infty} \psi\left(u_{i}\right)\right)^{1 / p}
\end{aligned}
$$

Hence,

$$
\sum_{i=1}^{\infty} \psi\left(u_{i}\right) \geq|z|^{p}\left(c_{1}^{-q / p} \sum_{i \in A_{1}}\left|x_{i}\right|^{q}+c_{2}^{-q / p} \sum_{i \in A_{2}}\left|x_{i}\right|^{q}\right)^{-p / q}
$$

Take

if $i \in A_{1}$, and

$$
u_{i}=-z\left(c_{1}^{-q / p} \sum_{i \in A_{1}}\left|x_{i}\right|^{q}+c_{2}^{-q / p} \sum_{i \in A_{2}}\left|x_{i}\right|^{q}\right)^{-1} c_{1}^{-q / p}\left|x_{i}\right|^{q / p}
$$

$$
u_{i}=z\left(c_{1}^{-q / p} \sum_{i \in A_{1}}\left|x_{i}\right|^{q}+c_{2}^{-q / p} \sum_{i \in A_{2}}\left|x_{i}\right|^{q}\right)^{-1} c_{2}^{-q / p}\left|x_{i}\right|^{q / p}
$$

if $i \in A_{2}$. Then, $\sum_{i=1}^{\infty} x_{i} u_{i}=z$ and

$$
\sum_{i=1}^{\infty} \psi\left(u_{i}\right)=|z|^{p}\left(c_{1}^{-q / p} \sum_{i \in A_{1}}\left|x_{i}\right|^{q}+c_{2}^{-q / p} \sum_{i \in A_{2}}\left|x_{i}\right|^{q}\right)^{-p / q}
$$

So,

$$
I(z)=|z|^{p}\left(c_{1}^{-q / p} \sum_{i \in A_{1}}\left|x_{i}\right|^{q}+c_{2}^{-q / p} \sum_{i \in A_{2}}\left|x_{i}\right|^{q}\right)^{-p / q} .
$$

The cases when either $z<0$ or $c_{1}=\infty$ or $c_{2}=\infty$ are similar. The case $1 \geq p>0$ follows similarly.

Next, we consider the LDP for series over a sequence of i.i.d.r.v.'s satisfying Condition (A.1). 
Theorem 3.2. Let $\left\{\xi_{k}\right\}$ be a sequence of i.i.d.r.v.'s with mean zero satisfying Condition (A.1) for some $p \geq 1$. Let $\left\{x_{k}\right\}_{k=1}^{\infty}$ be a sequence of real numbers such that $\sum_{k=1}^{\infty} x_{k}^{2}<\infty$. Let $X:=\sum_{k=1}^{\infty} x_{k} \xi_{k}$. Let $\epsilon_{n}:=(-\log (\mathbb{P}\{|\xi| \geq n\}))^{-1}$. Suppose that:

(i) For each $u>0$,

$$
\lim _{k \rightarrow \infty} \limsup _{n \rightarrow \infty} \epsilon_{n} \sum_{j=k+1}^{\infty} \Psi_{2}\left(u \epsilon_{n}^{-1} n^{-1} x_{j}\right)=0,
$$

where $\Phi(x)=-\log \left(\{\mathbb{P}\{|\xi| \geq|x|\}), x \in \mathbb{R}, \Psi(x)=\sup _{y \in \mathbb{R}}(x y-\Phi(y)), x \in \mathbb{R}\right.$, and $\Psi_{2}(t)=t^{2}$ for $|t| \leq 1$, and $\Psi_{2}(t)=\max (1, \Psi(t))$ for $|t|>1$.

(ii) If $p>2$, assume also that $\sum_{j=1}^{\infty}\left|x_{j}\right|^{q}<\infty$, where $\frac{1}{p}+\frac{1}{q}=1$.

Then, $\left\{n^{-1} X\right\}$ satisfies the LDP with speed $\epsilon_{n}^{-1}$ and rate function

$$
I(z)=\inf \left\{\sum_{j=1}^{\infty} \psi\left(u_{j}\right): \sum_{j=1}^{\infty} u_{j} x_{j}=z\right\},
$$

where $\psi$ is as in (3.1).

Proof. Let $X_{n, k}=n^{-1} \sum_{j=1}^{k} x_{j} \xi_{j}$ and let $X_{n}=n^{-1} \sum_{j=1}^{\infty} x_{j} \xi_{j}$. By Theorem 3.2 in Baxter and Jain [3], it suffices to prove that:

(C.1) For each $k \geq 1, X_{n, k}$, as $n \rightarrow \infty$, satisfies the LPD with speed $\epsilon_{n}^{-1}$ and rate function

$$
I_{k}(z)=\inf \left\{\sum_{j=1}^{k} \psi\left(u_{j}\right): \sum_{j=1}^{k} x_{j} u_{j}=z\right\} .
$$

(C.2) For each $\tau>0$,

$$
\lim _{k \rightarrow \infty} \limsup _{n \rightarrow \infty} \epsilon_{n} \log \left(\mathbb{P}\left\{\left|\sum_{j=k+1}^{\infty} x_{j} \xi_{j}\right| \geq \tau n\right\}\right)=-\infty .
$$

(C.3) For each $x \in \mathbb{R}$,

$$
I(x)=\lim _{\delta \rightarrow 0} \liminf _{k \rightarrow \infty} \inf \left\{I_{k}(y):|y-x| \leq \delta\right\} .
$$

By Lemma 2.8 in Lynch and Sethuraman [14] and Theorem 3.1, for each $k \geq 1,\left(n^{-1} \xi_{1}, \ldots, n^{-1} \xi_{k}\right)$ satisfies the LDP with speed $\epsilon_{n}^{-1}$ and rate function $\sum_{i=1}^{k} \psi\left(u_{i}\right)$. Hence, by the contraction principle for each $k \geq 1$, $\left\{n^{-1} \sum_{j=1}^{k} x_{j} \xi_{j}\right\}$ satisfies the LDP with speed $\epsilon_{n}^{-1}$ and rate function $I_{k}$. Hence (C.1) holds.

To check (C.2), we estimate the moment generating function of $\xi$. We claim that there exists a finite constant $c$ such that for each $\lambda \in \mathbb{R}$,

$$
\left|\log \left(E\left[\exp \left(\lambda \xi_{1}\right)\right]\right)\right| \leq c \Psi_{2}(2 \lambda) .
$$

Take $\lambda_{0}>0$ such that $\Psi\left(\lambda_{0}\right)>0$. Since $\xi_{1}$ has mean zero,

$$
E\left[\exp \left(\lambda \xi_{1}\right)-1\right]=O\left(\lambda^{2}\right), \text { as } \lambda \rightarrow 0 .
$$

This implies that there exists a constant $c$ such that (3.8) holds for each $|\lambda| \leq \lambda_{0}$.

Let $G$ be the df of $|\xi|$. Given $0<p<1$, let $G^{-1}(p)=\sup \{x \geq 0: G(x-) \leq p\}$. We have that for each $x \geq 0$ and each $0<p<1, G(x-) \leq p$ if an only if $x \leq G^{-1}(p)$. We also have that for each $0<p<1$, $G\left(G^{-1}(p)-\right) \leq p \leq G\left(G^{-1}(p)\right)$. We claim that each $0<a<1$,

$$
E\left[\exp \left(a \Phi\left(\xi_{1}\right)\right)\right] \leq(1-a)^{-1}
$$


We have that $\exp (-\Phi(x))=1-G(x-)$. So,

$$
\begin{aligned}
E\left[\exp \left(a \Phi\left(\xi_{1}\right)\right)\right] & =E\left[\left(1-G\left(\left|\xi_{1}\right|-\right)\right)^{-a}\right]=E\left[\int_{1-G\left(\left|\xi_{1}\right|-\right)}^{\infty} a t^{-a-1} \mathrm{~d} t\right] \\
& =E\left[\int_{0}^{\infty} I\left(1-G\left(\left|\xi_{1}\right|-\right)<t\right) a t^{-a-1} \mathrm{~d} t=\int_{0}^{\infty} P\left(1-t<G\left(\left|\xi_{1}\right|-\right)\right) a t^{-a-1} \mathrm{~d} t .\right.
\end{aligned}
$$

If $t>1, P\left(1-t<G\left(\left|\xi_{1}\right|-\right)\right)=1$. If $0<t<1$,

$$
\mathbb{P}\left\{1-t<G\left(\left|\xi_{1}\right|-\right)\right\}=\mathbb{P}\left\{G^{-1}(1-t)<\left|\xi_{1}\right|\right\}=1-G\left(G^{-1}(1-t)\right) \leq 1-(1-t)=t
$$

So,

$$
E\left[\exp \left(a \Phi\left(\xi_{1}\right)\right)\right] \leq \int_{0}^{1} a t^{-a} \mathrm{~d} t+\int_{1}^{\infty} a t^{-a-1} \mathrm{~d} t=(1-a)^{-1}
$$

and (3.9) follows. (3.9) implies that

$$
E\left[\exp \left(\left|\lambda \xi_{1}\right|\right)\right] \leq E\left[\exp \left(2^{-1} \Phi\left(\xi_{1}\right)+2^{-1} \Psi(2 \lambda)\right)\right] \leq 2 \exp \left(2^{-1} \Psi(2 \lambda)\right) .
$$

Hence, there exists a positive constant $c$ such that for each $|\lambda| \geq\left|\lambda_{0}\right|$,

$$
\log \left(E\left[\exp \left(\lambda \xi_{1}\right)\right]\right) \leq \log (2)+2^{-1} \Psi(2 \lambda) \leq c \Psi_{2}(2 \lambda)
$$

Therefore, (3.8) holds.

Given $\tau, u>0$,

$$
\begin{aligned}
\mathbb{P}\left\{\sum_{j=k+1}^{\infty} x_{j} \xi_{j} \geq \tau n\right\} & \leq \mathrm{e}^{-u \epsilon_{n}^{-1}} E\left[\exp \left(u \tau^{-1} n^{-1} \epsilon_{n}^{-1} \sum_{j=k+1}^{\infty} x_{j} \xi_{j}\right)\right] \\
& =\mathrm{e}^{-u \epsilon_{n}^{-1}} \prod_{j=k+1}^{\infty} E\left[\exp \left(u \tau^{-1} n^{-1} \epsilon_{n}^{-1} x_{j} \xi_{j}\right)\right] \\
& \leq \exp \left(-u \epsilon_{n}^{-1}+c \sum_{j=k+1}^{\infty} \Psi_{2}\left(2 u \tau^{-1} n^{-1} \epsilon_{n}^{-1} x_{j}\right)\right)
\end{aligned}
$$

Using a similar inequality for the lower bound, we get that

$$
\epsilon_{n} \log \left(\mathbb{P}\left\{\left|\sum_{j=k+1}^{\infty} x_{j} \xi_{j}\right| \geq \tau n\right\}\right) \leq \epsilon_{n} \log 2-u+\epsilon_{n} c \sum_{j=k+1}^{\infty} \Psi_{2}\left(2 u \tau^{-1} n^{-1} \epsilon_{n}^{-1} x_{j}\right) \rightarrow-u
$$

as $n \rightarrow \infty$. Hence, (C.2) holds.

(C.3) follows from Lemma 3.1.

The following condition is stronger than Condition (A.1):

(A.2) For some $p>0$ and some $0<b_{1}, b_{2} \leq \infty$ with $\min \left(b_{1}, b_{2}\right)<\infty$,

$$
\lim _{u \rightarrow \infty} u^{-p} \log (\mathbb{P}\{\xi \leq-u\})=-b_{1}
$$


and

$$
\lim _{u \rightarrow \infty} u^{-p} \log (\mathbb{P}\{\xi \geq u\})=-b_{2} .
$$

Under Condition (A.2), $\lim _{u \rightarrow \infty} u^{-p} \log (\mathbb{P}\{|\xi| \geq u\})=-b$, where $b=\min \left(b_{1}, b_{2}\right)$. Under Condition (A.2), if $\Phi(x)=-\log (\mathbb{P}\{|\xi| \geq|x|\}), \Psi(x)=\sup _{y \in \mathbb{R}}(x y-\Phi(y))$ and $p>1$, then

$$
\lim _{x \rightarrow \infty} x^{-q} \Psi(x) \rightarrow(b p)^{-q / p} q^{-1} .
$$

So, there are $0<c_{1}, c_{2}<\infty$ such that for $|x|$ large enough,

$$
c_{1}|x|^{q} \leq \Psi(x) \leq c_{2}|x|^{q} .
$$

Theorem 3.3. Let $\left\{\xi_{k}\right\}_{k=1}^{\infty}$ be a sequence of i.i.d.r.v.'s with mean zero satisfiying Condition (A.2) for some $p \geq 1$. Let $\left\{x_{k}\right\}_{k=1}^{\infty}$ be a sequence of real numbers such that $\sum_{k=1}^{\infty} x_{k}^{2}<\infty$. Let $X:=\sum_{k=1}^{\infty} x_{k} \xi_{k}$. Then,

(i) If $2 \geq p \geq 1$, then $\left\{n^{-1} X\right\}$ satisfies the LDP with speed $n^{p}$.

(ii) If $p>2$, then $\left\{n^{-1} X\right\}$ satisfies the LDP with speed $n^{p}$ and a good rate function if and only if $\sum_{j=1}^{\infty}\left|x_{j}\right|^{q}<\infty$, where $\frac{1}{p}+\frac{1}{q}=1$.

Moreover, the rate function is given by (3.1) with $c_{1}=b_{1}$ and $c_{2}=b_{2}$.

Proof. First let us prove (i). Assume that $2 \geq p>1$. We apply Theorem 3.2. There exists a finite constant $c$ such that for each $x \in \mathbb{R}, \Psi_{2}(x) \leq c\left(x^{2}+|x|^{q}\right)$. So, Hypothesis (i) in Theorem 3.2 holds. Let $u>0$. If $p=1$, for $k$ large $\left|u x_{j}\right| \leq 1$. So, for $k$ large enough,

$$
n^{-1} \sum_{j=k+1}^{\infty} \Psi_{2}\left(u x_{j}\right)=n^{-1} \sum_{j=k+1}^{\infty} u^{2} x_{j}^{2},
$$

and Hypothesis (i) in Theorem 3.2 holds.

Next, we prove (ii). Assume that $p>2$. There exists a finite constant $c$ such that for each $x \in \mathbb{R}$, $\Psi_{2}(x) \leq c|x|^{q}$. Hence, using Theorem 3.2, $\sum_{j=1}^{\infty}\left|x_{j}\right|^{q}<\infty$ implies that $\left\{n^{-1} X\right\}_{n=1}^{\infty}$ satisfies the LDP with speed $n^{p}$. Reciprocally, if $\left\{n^{-1} X\right\}_{n=1}^{\infty}$ satisfies the LDP with speed $n^{p}$ and a good rate function, then given $\tau>0$, there exists a $M>0$ such that for $n$ large enough,

$$
\mathbb{P}\left\{n^{-1}\left|\sum_{i=1}^{\infty} x_{i} \xi_{i}\right| \geq M\right\} \leq \mathrm{e}^{-\tau n^{p}} .
$$

Let $\left\{\xi_{i}^{\prime}\right\}$ be an independent copy of $\left\{\xi_{i}\right\}$. By symmetrization and the Lévy inequality, for each $N<\infty$,

$$
\begin{aligned}
\mathbb{P}\left\{n^{-1}\left|\sum_{i=1}^{N} x_{i}\left(\xi_{i}-\xi_{i}^{\prime}\right)\right| \geq 2 M\right\} & \leq 2 \mathbb{P}\left\{n^{-1}\left|\sum_{i=1}^{\infty} x_{i}\left(\xi_{i}-\xi_{i}^{\prime}\right)\right| \geq 2 M\right\} \\
& \leq 4 \mathbb{P}\left\{n^{-1}\left|\sum_{i=1}^{\infty} x_{i} \xi_{i}\right| \geq M\right\} \leq 4 \mathrm{e}^{-\tau n^{p}}
\end{aligned}
$$

So,

$$
\limsup _{n \rightarrow \infty} n^{-p} \log \left(\mathbb{P}\left\{n^{-1}\left|\sum_{i=1}^{N} x_{i}\left(\xi_{i}-\xi_{i}^{\prime}\right)\right| \geq 2 M\right\}\right) \leq-\tau
$$


for each $N<\infty$. By Theorem 3.2, $n^{-1} \sum_{i=1}^{N} x_{i}\left(\xi_{i}-\xi_{i}^{\prime}\right)$ satisfies the LDP with speed $n^{p}$ and rate function

$$
I(z)=\inf \left\{\sum_{i=1}^{N}\left(\psi\left(u_{i}\right)+\psi\left(v_{i}\right)\right): \sum_{i=1}^{N} x_{i}\left(u_{i}-v_{i}\right)=z\right\}
$$

where $\psi$ is as in (3.1) with $c_{i}=b_{i}$, for $i=1,2$. By Lemma 3.1 this rate function is

$$
I(z)=|z|^{p}\left(b_{1}^{-q / p}+b_{2}^{-q / p}\right)^{-p / q}\left(\sum_{i=1}^{N}\left|x_{i}\right|^{q}\right)^{-p / q}
$$

So,

$$
(2 M)^{p}\left(b_{1}^{-q / p}+b_{2}^{-q / p}\right)^{-p / q}\left(\sum_{i=1}^{N}\left|x_{i}\right|^{q}\right)^{-p / q} \geq \tau .
$$

Since this happens for each $N \geq 1, \sum_{i=1}^{\infty}\left|x_{i}\right|^{q}<\infty$.

One should expect that if $p>2$ and $\sum_{i=1}^{\infty}\left|x_{i}\right|^{p /(p-1)}=\infty$, then $\left\{n^{-1} X\right\}$ still satisfies a LDP, but with smaller speed. Next theorem presents a situation where this happens:

Theorem 3.4. Let $\left\{\xi_{k}\right\}_{k=1}^{\infty}$ be a sequence of symmetric i.i.d.r.v.'s such that $\mathbb{P}\left\{\left|\xi_{1}\right| \geq t\right\}=\exp \left(-c^{p}\right)$, for each $t>0$, where $p>2$ and $c>0$. Let $2^{-1}<b<(p-1) / p$. Then,

$$
\lim _{t \rightarrow \infty} t^{-1 /(1-b)} \log \left(\mathbb{P}\left\{\left|\sum_{j=1}^{\infty} j^{-b} \xi_{j}\right| \geq t\right\}\right)=-(1-b) b^{b /(b-1)} a^{-b /(b-1)}
$$

where $a:=\int_{0}^{\infty} \ln E\left[\exp \left(x^{-b} \xi\right)\right] \mathrm{d} x$.

Proof. Let $X:=\sum_{j=1}^{\infty} j^{-b} \xi_{j}$. Let $\psi(\lambda)=\log E\left[\mathrm{e}^{\lambda \xi}\right]$. By $(3.8), \psi(\lambda)=O\left(\min \left(\lambda^{2},|\lambda|^{q}\right)\right)$. This estimation and the restriction $2^{-1}<b<(p-1) / p$ imply that for each $\lambda>0$,

$$
\int_{0}^{\infty} \psi\left(x^{-b} \lambda\right) \mathrm{d} x<\infty
$$

So, $0<a<\infty$.

It suffices to show that $\left\{n^{-1} \sum_{j=1}^{\infty} j^{-b} \xi_{j}\right\}$ satisfies the LPD with speed $n^{1 /(1-b)}$ and rate function $I(t)=$ $|t|^{1 /(1-b)}(1-b) b^{b /(b-1)} a^{-b /(b-1)}$. By the Gärtner-Ellis theorem (see for example Th. 2.3.2 in Dembo and Zeitouni $[6])$, it suffices to prove that for each $\lambda \in \mathbb{R}$,

$$
n^{-1 /(1-b)} \ln E\left[\exp \left(\lambda n^{1 /(1-b)} n^{-1} X\right)\right] \rightarrow|\lambda|^{1 / b} a
$$

and that

$$
I(t)=\sup _{\lambda \in R}\left(\lambda t-|\lambda|^{1 / b} a\right)=(1-b) b^{b /(b-1)} a^{-b /(b-1)}|t|^{1 /(b-1)} .
$$


We have that

$$
\begin{aligned}
n^{-1 /(1-b)} \ln E\left[\exp \left(\lambda n^{1 /(1-b)} n^{-1} X\right)\right] & =n^{-1 /(1-b)} \ln E\left[\exp \left(\lambda n^{b /(1-b)} \sum_{j=1}^{\infty} j^{-b} \xi_{j}\right)\right] \\
& =n^{-1 /(1-b)} \sum_{j=1}^{\infty} \psi\left(n^{b /(1-b)} j^{-b} \lambda\right) \\
& =\sum_{j=1}^{\infty} \int_{(j-1) n^{-1 /(1-b)}}^{j n^{-1 /(1-b)}} \psi\left(\left(j n^{-1 /(1-b)}\right)^{-b} \lambda\right) \mathrm{d} x \\
& \rightarrow \int_{0}^{\infty} \psi\left(x^{-b} \lambda\right) \mathrm{d} x=|\lambda|^{1 / b} a
\end{aligned}
$$

by the monotone convergence theorem (using that $\psi$ is even and increasing in $(0, \infty)$ ). Hence, the claim follows.

In the previous theorem, $n^{1 /(1-b)} \ll n^{p}$ and the tail of $\left|\sum_{j=1}^{\infty} j^{-b} \xi_{j}\right|$ is asymptotically much bigger than the tail of $\xi_{1}$.

Finally, we consider stochastic processes whose underlying r.v.'s satisfy Condition (A.1). We present sufficient conditions for the LDP of certain stochastic processes.

Theorem 3.5. Let $\left\{\xi_{k}\right\}$ be a sequence of symmetric i.i.d.r.v.'s with satisfying Condition (A.1) for some $p \geq 1$ and Condition (B.1). Let $T$ be a parameter set. Let d be a pseudometric in T. Let $X(t):=\sum_{k=1}^{\infty} x_{k}(t) \xi_{k}$, where $\sum_{k=1}^{\infty} x_{k}^{2}(t)<\infty$. Suppose that:

(i) For each $u>0$ and each $t \in T$,

$$
\lim _{k \rightarrow \infty} \limsup _{n \rightarrow \infty} \epsilon_{n} \sum_{j=k+1}^{\infty} \Psi_{2}\left(u \epsilon_{n}^{-1} n^{-1} x_{j}(t)\right)=0,
$$

where $\Psi_{2}$ is as in Theorem 3.2.

(ii) If $p>2$, assume also that for each $t \in T, \sum_{j=1}^{\infty}\left|x_{j}(t)\right|^{q}<\infty$, where $\frac{1}{p}+\frac{1}{q}=1$.

(iii) $\sup _{t \in T}|X(t)|<\infty$ a.s.

(iv) $(T, d)$ is totally bounded.

(v) $\left(T, d_{q}\right)$ is totally bounded, where $d_{q}(s, t)=\|x(s)-x(t)\|_{q}$.

(vi) For each $u>0$,

$$
\lim _{\eta \rightarrow 0} \limsup _{n \rightarrow \infty} n^{-1} L_{\Phi}\left(u \epsilon_{n}^{-1}, \eta\right)=0
$$

where

$$
L_{\Phi}(u, \eta)=: \sup \left\{\sum_{k=1}^{\infty}\left|x_{k}(s)-x_{k}(t)\right|\left(\left|a_{k}\right|+\left|b_{k}\right|\right): \sum_{k=1}^{\infty}\left(\Phi\left(a_{k}\right)\right)^{2} \leq 36 u, \sum_{k=1}^{\infty} \Phi\left(b_{k}\right) \leq 9 u, d(s, t) \leq \eta\right\} .
$$

Then, $\left\{n^{-1} X(t): t \in T\right\}$ satisfies the LDP in $l_{\infty}(T)$ with speed $\epsilon_{n}^{-1}$ and rate function

$$
I(z)=\inf \left\{\sum_{i=1}^{\infty} \psi\left(\gamma_{i}\right): \sum_{i=1}^{\infty} x_{i}(t) \gamma_{i}=z(t) \text { for each } t \in T\right\}
$$

where $\psi$ is as in (3.1). 
Proof. We apply Theorem 3.1 in Arcones [1]. Conditions (i) and (ii) imply the LDP for the finite dimensional distributions. We need to prove that for each $\tau>0$,

$$
\lim _{\delta \rightarrow 0} \limsup _{n \rightarrow \infty} \epsilon_{n} \log \left(\mathbb{P}\left\{\sup _{d(s, t) \leq \eta}|X(s)-X(t)| \geq n \tau\right\}\right)=-\infty .
$$

Theorem 2.2 and Conditions (iii) and (vi) imply (3.11).

To prove that the rate function is as claimed, we apply Theorem 4.2 in Arcones [2] with $S=\{1,2, \ldots\}, \psi$ as in (3.1) and $\mu$ equal to the counting measure. To apply this theorem, we need that for each integer $k \geq 1$, $\left(T, \rho_{k}\right)$ is totally bounded where

$$
\rho_{k}(s, t):=\sup \left\{\left|\sum_{j=1}^{\infty}\left(x_{j}(s)-x_{j}(t)\right) \gamma_{j}\right|: \sum_{j=1}^{\infty} \psi\left(\gamma_{j}\right) \leq k\right\} .
$$

This follows from Condition (v).

When Condition (A.2) is satisfied, we get necessary and sufficient conditions for the LDP:

Corollary 3.1. Let $\left\{\xi_{k}\right\}$ be a sequence of symmetric i.i.d.r.v.'s with mean zero satisfying Condition (B.1), $\tau:=\inf _{x>0} x^{-1} \Phi(x)>0$, and that

$$
\lim _{u \rightarrow \infty} u^{-p} \log (\mathbb{P}\{|\xi| \geq u\})=-b
$$

for some $p \geq 1$ and some $b>0$. Let $T$ be a parameter set. Let $X(t):=\sum_{k=1}^{\infty} x_{k}(t) \xi_{k}$, where $\sum_{i=1}^{\infty}\left|x_{i}(t)\right|^{2}<\infty$. Then, the following sets of conditions are equivalent:

(a.1) For each $t \in T,\left(x_{i}(t)\right)_{i=1}^{\infty} \in l_{q}$.

(a.2) $\left(T, d_{q}\right)$ is totally bounded, where $d_{q}(s, t)=\|x(s)-x(t)\|_{q}$.

(a.3) $\sup _{t \in T}|X(t)|<\infty$ a.s.

(b) $\left\{n^{-1} X(t): t \in T\right\}$ satisfies the $L D P$ in $l_{\infty}(T)$ with speed $n^{p}$ and a good rate.

Moreover, the rate function is

$$
I(z)=\inf \left\{\sum_{i=1}^{\infty} \psi\left(\gamma_{i}\right): \sum_{i=1}^{\infty} x_{i}(t) \gamma_{i}=z(t) \text { for each } t \in T\right\}
$$

where $\psi$ is as in (3.1) with $c_{1}=c_{2}=b$.

Proof. Assume (a). We apply Theorem 3.5 with $d(s, t)=\|\left(x(s)-x(t) \|_{q}\right.$. Hypothesis (i) in Theorem 3.5 follows from an argument in Theorem 3.3. It is easy to see that Conditions (ii)-(v) in Theorem 3.5 hold. As to Hypothesis (vi) in Theorem 3.5. By (2.3) and (2.4), if $p \geq 2$,

$$
n^{-1} L_{\Phi}\left(u n^{p}, \eta\right) \leq \eta\left(36 \tau^{2} u\right)^{1 / p}+\eta(9 \tau u)^{1 / p}
$$

and if $1 \leq p<2$,

$$
n^{-1} L_{\Phi}\left(u n^{p}, \eta\right) \leq 12 \sup _{t \in T}\|x(t)\|_{2} \tau u^{1 / 2} n^{(p-2) / 2}+\eta(9 \tau u)^{1 / p} .
$$

Therefore, Hypothesis (vi) in Theorem 3.5 holds. 
Assume (b). Theorem 3.3 (ii) implies (a.1). By Theorem 3.1 in Arcones [1], for each positive integer $k$, $\left(T, \rho_{k}\right)$ is totally bounded, where

$$
\begin{aligned}
\rho_{k}(s, t) & =\sup \left\{\left|u_{2}-u_{1}\right|: I_{s, t}\left(u_{1}, u_{2}\right) \leq k\right\} \\
& =\sup \left\{\left|\sum_{j=1}^{\infty}\left(x_{j}(t)-x_{j}(s)\right) \gamma_{j}\right|: \sum_{j=1}^{\infty} \psi\left(\gamma_{j}\right) \leq k\right\} \\
& =\sup \left\{\left|\sum_{j=1}^{\infty}\left(x_{j}(t)-x_{j}(s)\right) \gamma_{j}\right|: \sum_{j=1}^{\infty} b\left|\gamma_{j}\right|^{p} \leq k\right\}=\|x(t)-x(s)\|_{q} p^{-1 / p} k^{1 / p}
\end{aligned}
$$

and $I_{s, t}$ is the rate of the LDP of $\left(n^{-1} X(s), n^{-1} X(t)\right)$. This implies (a.2).

Since $\left\{z \in l_{\infty}(T): I(z) \leq 1\right\}$ is a compact set, there exists $0<c<\infty$ such that

$$
\left\{z \in l_{\infty}(T): I(z) \leq 1\right\} \subset\left\{z \in l_{\infty}(T):|z|_{\infty} \leq c\right\} .
$$

So,

Hence, for $n$ large enough

$$
\limsup _{n \rightarrow \infty} n^{-p} \log \left(\mathbb{P}\left\{n^{-1} \sup _{t \in T}\left|\sum_{i=1}^{\infty} x_{i}(t) \xi_{i}\right| \geq c\right\}\right) \leq-1 .
$$

$$
\mathbb{P}\left\{n^{-1} \sup _{t \in T}\left|\sum_{i=1}^{\infty} x_{i}(t) \xi_{i}\right| \geq c\right\} \leq \exp \left(-2^{-1} n^{p}\right) .
$$

Hence, (a.3) follows.

Next, we consider the LDP when Condition (A.2) holds for some $0<p \leq 1$.

Theorem 3.6. Let $\left\{\xi_{k}\right\}$ be a sequence of symmetric i.i.d.r.v.'s with mean zero satisfying Condition (B.2) and that

$$
\lim _{u \rightarrow \infty} u^{-p} \log (\mathbb{P}\{|\xi| \geq u\})=-b
$$

for some $1 \geq p>0$ and some $b>0$. Let $X=\sum_{k=1}^{\infty} x_{k} \xi_{k}$. Then, $\left\{n^{-1} X\right\}$ satisfies the LDP with speed $n^{p}$ and rate function

$$
I(z)=\inf \left\{\sum_{j=1}^{\infty} \psi\left(u_{j}\right): \sum_{j=1}^{\infty} u_{j} x_{j}=z\right\},
$$

where $\psi$ is as in (3.1) with $c_{1}=c_{1}=b$.

Proof. We proceed as in the proof of Theorem 3.2, applying Theorem 3.1 in Baxter and Jain [3] with $X_{n, k}=$ $n^{-1} \sum_{j=1}^{k} x_{j} \xi_{j}$ and $X_{n}=n^{-1} \sum_{j=1}^{\infty} x_{j} \xi_{j}$.

By Lemma 2.8 in Lynch and Sethuraman [14], and the contraction principle, for each $k \geq 1,\left\{n^{-1} \sum_{j=1}^{k} x_{j} \xi_{j}\right\}$ satisfies the LDP with speed $\epsilon_{n}^{-1}$ and rate function

$$
I_{k}(z)=\inf \left\{\sum_{j=1}^{k} \psi\left(u_{j}\right): \sum_{j=1}^{k} x_{j} u_{j}=z\right\} .
$$

We need to prove that for each $\tau>0$,

$$
\lim _{k \rightarrow \infty} \limsup _{n \rightarrow \infty} n^{-p} \log \left(\mathbb{P}\left\{\left|\sum_{j=k+1}^{\infty} x_{j} \xi_{j}\right| \geq \tau n\right\}\right)=-\infty .
$$


Theorem 2.3 implies that for $n$ large enough,

$$
\mathbb{P}\left\{\left|\sum_{i=k+1}^{\infty}\left(x_{i}-x_{i}\right)\left(\xi_{i}-\xi_{i}^{\prime}\right)\right| \geq 2^{-1} n \tau\right\} \leq 4 \exp \left(-c n^{p}\left(\sup _{j \geq k+1}\left|x_{j}\right|\right)^{-p}\right)
$$

Hence, (3.12) holds.

Next, we consider the LDP for stochastic processes over a sequence of i.i.d.r.v.'s satisfying the conditions in the previous theorem.

Theorem 3.7. Let $\left\{\xi_{k}\right\}$ be a sequence of symmetric i.i.d.r.v.'s with mean zero satisfying Condition (B.2) and that

$$
\lim _{u \rightarrow \infty} u^{-p} \log (\mathbb{P}\{|\xi| \geq u\})=-b
$$

for some $1 \geq p>0$ and some $b>0$. Let $T$ be a parameter set. Let $X(t):=\sum_{k=1}^{\infty} x_{k}(t) \xi_{k}$, where $\sum_{i=1}^{\infty}\left|x_{i}(t)\right|^{2}<$ $\infty$ for each $t \in T$. Then, the following sets of conditions are equivalent:

(a.1) $(T, d)$ is totally bounded, where $d(s, t)=\sup _{i \geq 1}\left|x_{i}(s)-x_{i}(t)\right|$.

(a.2) $\sup _{t \in T}\left|\sum_{k=1}^{\infty} x_{k}(t) \xi_{k}\right|<\infty$ a.s.

(b) $\left\{n^{-1} X(t): t \in T\right\}$ satisfies the LDP in $l_{\infty}(T)$ with speed $n^{1 / p}$.

Moreover, the rate function is

$$
I(z)=\inf \left\{\sum_{j=1}^{\infty} \psi\left(\gamma_{j}\right): \sum_{j=1}^{\infty} x_{j}(t) \gamma_{j}=z(t) \text { for each } t \in T\right\}
$$

where $\psi$ is as in (3.1) with $c_{1}=c_{2}=b$.

Proof. Assume (a). We apply Theorem 3.1 in Arcones [1]. Theorem 3.5 implies the LDP for the finite dimensional distributions. Theorem 2.3 implies that for each $\tau>0$,

$$
\lim _{\delta \rightarrow 0} \limsup _{n \rightarrow \infty} n^{-p} \log \left(\mathbb{P}\left\{\sup _{d(s, t) \leq \eta}\left|\sum_{i=1}^{\infty}\left(x_{i}(s)-x_{i}(t)\right) \xi_{i}\right| \geq n \tau\right\}\right)=-\infty .
$$

Assume (b). By Theorem 3.1 in Arcones [1], $(T, \rho)$ is totally bounded, where

$$
\rho_{k}(s, t)=\sup \left\{\left|u_{2}-u_{1}\right|: I_{s, t}\left(u_{1}, u_{2}\right) \leq k\right\}
$$

and

$$
I_{s, t}\left(u_{1}, u_{2}\right)=\left\{\sum_{j=1}^{\infty} \phi\left(\gamma_{j}\right): \sum_{j=1}^{\infty} x_{j}(s) \gamma_{j}=u_{1}, \sum_{j=1}^{\infty} x_{j}(t) \gamma_{j}=u_{2}\right\} .
$$

It is easy to see that $\rho_{k}(s, t) \geq c k^{-1 / p}|x(s)-x(t)|_{\infty}$, where $c$ is positive constant. This implies (a.1).

The LDP implies that there are positive constants $r, M$ such that for $n$ large enough,

$$
n^{-p} \log \left(\mathbb{P}\left\{\sup _{t \in T}|X(t)| \geq n r\right) \leq-M\right.
$$

This implies that for some $c>0$,

In particular (a.2) holds.

$$
E\left[\exp \left(c \sup _{t \in T}|X(t)|^{p}\right)\right]<\infty .
$$




\section{REFERENCES}

[1] M.A. Arcones, The large deviation principle for stochastic processes I. Theor. Probab. Appl. 47 (2003) 567-583.

[2] M.A. Arcones, The large deviation principle for stochastic processes. II. Theor. Probab. Appl. 48 (2004) 19-44.

[3] J.R. Baxter and C.J. Naresh, An approximation condition for large deviations and some applications, in Convergence in ergodic theory and probability (Columbus, OH, 1993), de Gruyter, Berlin. Ohio State Univ. Math. Res. Inst. Publ. 5 (1996) 63-90.

[4] N.H. Bingham, C.M. Goldie and J.L. Teugels, Regular Variation. Cambridge University Press, Cambridge, UK (1987).

[5] Y.S. Chow and H. Teicher, Probability Theory. Independence, Interchangeability, Martingales. Springer-Verlag, New York (1978).

[6] A. Dembo and O. Zeitouni, Large Deviations Techniques and Applications. Springer, New York (1998).

[7] J.D. Deuschel and D.W. Stroock, Large Deviations. Academic Press, Inc., Boston, MA (1989).

[8] E.D. Gluskin and S. Kwapień, Tail and moment estimates for sums of independent random variables with logarithmically concave tails. Studia Math. 114 (1995) 303-309.

[9] P. Hitczenko, S.J. Montgomery-Smith and K. Oleszkiewicz, Moment inequalities for sums of certain independent symmetric random variables. Studia Math. 123 (1997) 15-42.

[10] S. Kwapień and W.A. Woyczyński, Random Series and Stochastic Integrals: Single and Multiple. Birkhäuser, Boston (1992).

[11] R. Latala, Tail and moment estimates for sums of independent random vectors with logarithmically concave tails. Studia Math. 118 (1996) 301-304.

[12] M. Ledoux and M. Talagrand, Probability in Banach Spaces. Springer-Verlag, New York (1991).

[13] M. Ledoux, The Concentration of Measure Phenomenon. American Mathematical Society, Providence, Rhode Island (2001).

[14] J. Lynch and J. Sethuraman, Large deviations for processes with independent increments. Ann. Probab. 15 (1987) 610-627.

[15] M. Talagrand, A new isoperimetric inequality and the concentration of measure phenomenon. Geometric aspects of functional analysis (1989-90), Springer, Berlin. Lect. Notes Math. 1469 (1991) 94-124.

[16] M. Talagrand, The supremum of some canonical processes. Amer. J. Math. 116 (1994) 283-325.

[17] S.R.S. Varadhan, Asymptotic probabilities and differential equations. Comm. Pures App. Math. 19 (1966) 261-286. 